\title{
Orientation phenomena for electron-ion collisional excitations in a classical nonideal plasma
}

\author{
Y O UNG-D A E J U N G ${ }^{1,2}$ \\ ${ }^{1}$ Department of Physics, 0319, University of California, San Diego, 9500 Gilman Drive, \\ La Jolla, CA 92093-0319, USA \\ ${ }^{2}$ Permanent address: Department of Physics, Hanyang University \\ Ansan, Kyunggi-Do 425-791, South Korea \\ (ydjung@physics.ucsd.edu; yjung@bohr.hanyang.ac.kr)
}

(Received 24 May 2001 and in revised form 21 September 2001)

\begin{abstract}
Orientation phenomena for direct $1 s \rightarrow 2 p_{ \pm 1}$ excitations in electronhydrogenic-ion collisions are investigated in a classical nonideal plasma. An effective pseudopotential model taking into account plasma-screening and collective effects is applied to describe the interaction potential in a nonideal plasma. The orientation parameter for direct $1 s \rightarrow 2 p_{ \pm 1}$ excitations in a nonideal plasma is obtained as a function of the impact parameter, the nonideal-plasma parameter, the Debye length, and the projectile energy. The result shows that the nonideal effect reduces the propensity of the $1 s \rightarrow 2 p_{-1}$ transition. It is also found that the nonideal effect on the orientation parameter increases with increasing projectile energy.
\end{abstract}

\section{Introduction}

Electron-ion collisions (Janev et al. 1985; Jung 1995a; Kobzev et al. 1995; Balashov et al. 2000) in plasmas have been extensively investigated in recent years, since these processes can be used for plasma diagnostics. Orientation and alignment phenomena in electron-atom and electron-ion collisions have been actively studied, since these phenomena provide detailed information on the mechanism of collisional excitation of target atoms and ions (McDowell and Coleman 1970). An experimental investigation has shown the possibility of the detection of radiative transitions from the excited $p_{ \pm 1}(m= \pm 1)$ states to the $1 s$ ground state (Andersen et al. 1990). Orientation phenomena in plasmas could provide detailed information about plasma parameters. Recently, orientation phenomena for $s-p$ electron-ion excitations in weakly coupled plasmas (Jung 1995b; Jung and Cho 1995) have been investigated using the nonspherical Debye-Hückel model potential. The Debye-Hückel potential describes the properties of a low-density plasma and corresponds to a pair-correlation approximation. A plasma described by the Debye-Hückel model is called an ideal plasma, since the average energy of interaction between particles is small compared with the average kinetic energy of a particle. However, multiparticle correlation effects caused by simultaneous interaction of many particles should be taken into account with increasing plasma density. Then, it is necessary to take into account not only short-range collective effects 
but also long-range effects in the case of a plasma with a moderate density and temperature. In this case, the interaction potential cannot be described by the Debye-Hückel model owing to the strong collective effects of nonideal particle interaction (Baimbetov et al. 1995; Arkhipov and Davletov 1998; Arkhipov et al. $2000 \mathrm{a}, \mathrm{b})$. Thus, orientation phenomena for electron-ion excitations in a nonideal plasma will be different from those in an ideal plasma. Therefore, in this paper, we investigate plasma-screening and collective effects on orientation phenomena for the $1 s \rightarrow 2 p_{ \pm 1}$ transitions of the hydrogenic-ion target in a classical nonideal plasma. A pseudopotential model including plasma-screening and collective effects is applied to describe the interaction potential between the projectile electron and the target ion in a nonideal plasma. We use the semiclassical straight-line trajectory method (Janev et al. 1985) to describe the motion of the high-energy projectile electron and to visualize the plasma-screening and collective effects on the orientation parameter. The orientation parameter for direct $1 s \rightarrow 2 p_{ \pm 1}$ excitations is obtained as a function of the impact parameter, the nonideal-plasma parameter, the Debye length, and the energy of the projectile electron.

In Sec. 2, we derive a closed form of the semiclassical transition amplitude for direct $1 s \rightarrow 2 p_{ \pm 1}$ excitations in electron-hydrogenic-ion collisions in a nonideal plasma using the pseudopotential model including plasma-screening and collective effects with the semiclassical path approximation. In Sec. 3, we obtain the orientation parameter for direct $1 s \rightarrow 2 p_{ \pm 1}$ excitations as a function of the impact parameter, the nonideal-plasma parameter, the Debye length, and the projectile energy. We also investigate plasma-screening and collective effects on the orientation parameter and the propensity rule with changing nonideal-plasma parameter. Finally, in Sec. 4, a summary and discussion are given.

\section{Transition amplitude}

From the first-order semiclassical approximation, the cross-section (Jung 1993) for excitation from an unperturbed atomic state $|n\rangle\left(\equiv \Psi_{n l m}(\mathbf{r})\right)$ to an excited state $\left|n^{\prime}\right\rangle\left(\equiv \Psi_{n^{\prime} l^{\prime} m^{\prime}}(\mathbf{r})\right)$ is given by

$$
\sigma_{n^{\prime}, n}=\int d^{2} \mathbf{b}\left|T_{n^{\prime}, n}(\mathbf{b})\right|^{2},
$$

where $\mathbf{b}$ is the impact parameter and $T_{n^{\prime}, n}(\mathbf{b})$ is the transition amplitude,

$$
T_{n^{\prime}, n}=-\frac{i}{\hbar} \int_{-\infty}^{\infty} d t e^{i \omega_{n^{\prime}, n} t}\left\langle n^{\prime}|V(\mathbf{R}, \mathbf{r})| n\right\rangle
$$

where $\omega_{n^{\prime}, n}\left(\equiv\left(E_{n^{\prime}}-E_{n}\right) / \hbar\right)$ is the transition frequency, and $E_{n}$ and $E_{n^{\prime}}$ are the energies of atomic states $n$ and $n^{\prime}$, respectively. Here, $V(\mathbf{R}, \mathbf{r})$ is the interaction potential between the projectile electron and the target ion, and $\mathbf{R}$ and $\mathbf{r}$ are, respectively, the positions of the projectile electron and a bound electron in the target system. This semiclassical impact-parameter method has a strong appeal in aiding physical intuition, since calculations based on this method are mathematically more tractable than fully quantum-mechanical treatments.

In Baimbetov et al. (1995), an integro-differential equation for the effective potential of the particle interaction, taking into account the simultaneous correlations of many particles, was obtained on the basis of a sequential solution of the chain of Bogolyubov equations for the equilibrium distribution function of particles of a 
classical nonideal plasma, and an analytical expression for the pseudopotential of the particle interaction in a nonideal plasma was also obtained by application of the spline approximation. For the sake of simplicity, we assume that the target is the hydrogenic ion with nuclear charge $Z$. The interaction potential $V(\mathbf{R}, \mathbf{r})$ between the electron and the target ion in a nonideal plasma using the pseudopotential, taking into account plasma-screening and collective effects, can be obtained as

$$
\begin{aligned}
V(\mathbf{R}, \mathbf{r})= & -\frac{Z e^{2}}{R} e^{-R / \Lambda} \frac{1+\frac{1}{2} \gamma f(R / \Lambda)}{1+c(\gamma)} \\
& +\frac{e^{2}}{|\mathbf{R}-\mathbf{r}|} e^{-|\mathbf{R}-\mathbf{r}| / \Lambda} \frac{1+\frac{1}{2} \gamma f(|\mathbf{R}-\mathbf{r}| / \Lambda)}{1+c(\gamma)}
\end{aligned}
$$

where $\Lambda$ is the Debye length, $\gamma\left(\equiv e^{2} / \Lambda k T_{e}\right)$ is the nonideal-plasma parameter, $f(x / \Lambda)=\frac{1}{5}\left(e^{-\sqrt{\gamma} x / \Lambda}-1\right)\left(1-e^{-2 x / \Lambda}\right), c(\gamma) \approx 0.456 \gamma-0.108 \gamma^{2}$ is the correlation coefficient, and $T_{e}$ is the electron temperature. When $\gamma \rightarrow 0$, i.e., for a weakly nonideal plasma, the pseudopotential (3) goes over to the nonspherical DebyeHückel interaction potential:

$$
V_{D H}(\mathbf{R}, \mathbf{r}) \rightarrow-\frac{Z e^{2}}{R} e^{-R / \Lambda}+\frac{e^{2}}{|\mathbf{R}-\mathbf{r}|} e^{-|\mathbf{R}-\mathbf{r}| / \Lambda} .
$$

For inelastic scattering $\left(n \neq n^{\prime}\right)$, the projectile-electron-target-nucleus interaction term (the first term in (3)) does not contribute to the transition amplitude $T_{n^{\prime}, n}$ only to the orthogonality of the initial and final states of the target system, i.e., $\left\langle n^{\prime} \mid n\right\rangle=\delta_{n^{\prime}, n} \delta_{l^{\prime}, l} \delta_{m^{\prime}, m}$. Thus, the transition-matrix element is then

$$
\begin{aligned}
\left\langle n^{\prime}|V(\mathbf{R}, \mathbf{r})| n\right\rangle & =e^{2}\left\langle n^{\prime}\left|\frac{e^{-|\mathbf{R}-\mathbf{r}| / \Lambda}}{|\mathbf{R}-\mathbf{r}|} \frac{1+\frac{1}{2} \gamma f(|\mathbf{R}-\mathbf{r}| / \Lambda)}{1+c(\gamma)}\right| n\right\rangle, \\
& \equiv e^{2} \bar{V}_{n^{\prime}, n} .
\end{aligned}
$$

Then, for $1 s \rightarrow 2 p_{ \pm 1}$ excitations, the transition-matrix elements $\bar{V}_{2 p_{ \pm 1}, 1 s}$, are given by

$$
\bar{V}_{2 p_{ \pm 1}, 1 s}=\int d^{3} \mathbf{r} \Psi_{2 p_{ \pm 1}}^{*}(\mathbf{r}) \frac{e^{-|\mathbf{R}-\mathbf{r}| / \Lambda}}{|\mathbf{R}-\mathbf{r}|} \frac{1+\frac{1}{2} \gamma f(|\mathbf{R}-\mathbf{r}| / \Lambda)}{1+c(\gamma)} \Psi_{1 s}(\mathbf{r}) .
$$

Since the nonspherical electron-electron interaction term $\left(e^{-\alpha|\mathbf{R}-\mathbf{r}|} /|\mathbf{R}-\mathbf{r}|\right)$ has the form of the modified Helmholtz operator Green function, it can be expanded using the addition theorem with spherical harmonics $Y_{l m}$. For large $R$, the modified Helmholtz operator Green function can be represented as

$$
\frac{e^{-\alpha|\mathbf{R}-\mathbf{r}|}}{|\mathbf{R}-\mathbf{r}|} \cong 4 \pi \alpha \sum_{l=0}^{\infty} \sum_{m=-l}^{l} k_{l}(\alpha R) i_{l}(\alpha r) Y_{l m}^{*}(\hat{R}) Y_{l m}(\hat{r}),
$$

where $k_{l}(\alpha R)$ and $i_{l}(\alpha r)$ are the spherical modified Bessel functions (Arfken and Weber 2000) in Rayleigh's representation:

$$
\begin{gathered}
k_{l}(x)=(-1)^{l} x^{l}\left(\frac{1}{x} \frac{d}{d x}\right)^{l} \frac{e^{-x}}{x} \\
i_{l}(x)=x^{l}\left(\frac{1}{x} \frac{d}{d x}\right)^{l} \frac{\sinh x}{x} .
\end{gathered}
$$


Then, the $1 s \rightarrow 2 p_{ \pm 1}$ transition-matrix elements are obtained as follows:

$$
\begin{aligned}
\bar{V}_{2 p_{ \pm 1}, 1 s}= & \frac{\sqrt{4 \pi}}{1+c(\gamma)} \frac{Y_{1 \pm 1}^{*}(\hat{R})}{\Lambda} \\
& \times\left\{k_{1}\left(\frac{R}{\Lambda}\right) \int_{0}^{R} r^{2} d r R_{2 p}(r) i_{1}\left(\frac{r}{\Lambda}\right) R_{1 s}(r)\right. \\
& +\frac{\gamma}{5}\left[(\sqrt{\gamma}+1) k_{1}\left(\frac{\sqrt{\gamma}+1}{\Lambda} R\right) \int_{0}^{R} r^{2} d r R_{2 p}(r) i_{1}\left(\frac{\sqrt{\gamma}+1}{\Lambda} R\right) R_{1 s}(r)\right. \\
& -(\sqrt{\gamma}+3) k_{1}\left(\frac{\sqrt{\gamma}+3}{\Lambda} R\right) \int_{0}^{R} r^{2} d r R_{2 p}(r) i_{1}\left(\frac{\sqrt{\gamma}+3}{\Lambda} R\right) R_{1 s}(r) \\
& -k_{1}\left(\frac{R}{\Lambda}\right) \int_{0}^{R} r^{2} d r R_{2 p}(r) i_{1}\left(\frac{R}{\Lambda}\right) R_{1 s}(r) \\
& \left.\left.+3 k_{1}\left(\frac{3}{\Lambda} R\right) \int_{0}^{R} r^{2} d r R_{2 p}(r) i_{1}\left(\frac{3}{\Lambda} R\right) R_{1 s}(r)\right]\right\}
\end{aligned}
$$

where $R_{1 s}(r)$ and $R_{2 p}(r)$ are the radial $1 s$ and $2 p$ atomic wave functions (Bethe and Salpeter 1957), respectively:

$$
\begin{aligned}
& R_{1 s}(r)=2 a_{Z}^{-3 / 2} e^{-r / a_{Z}} \\
& R_{2 p}(r)=\frac{1}{2 \sqrt{6}} a_{Z}^{-5 / 2} r e^{-r / 2 a_{Z}}
\end{aligned}
$$

here $a_{Z}\left(\equiv a_{0} / Z=\hbar^{2} / Z m e^{2}\right)$ is the Bohr radius of the hydrogenic ion with nuclear charge $Z$. After some algebra, the $1 s \rightarrow 2 p_{ \pm 1}$ transition matrix elements are then found to be

$$
\begin{aligned}
\bar{V}_{2 p_{ \pm 1}, 1 s}= & \frac{4 \sqrt{6 \pi}}{1+c(\gamma)} \frac{Y_{1 \pm 1}^{*}(\hat{R})}{a_{Z}}\left\{\frac{1}{\left(\frac{9}{4}-a_{\Lambda}^{2}\right)^{3}}\left(\frac{1}{\bar{R}^{2}}+\frac{a_{\Lambda}}{\bar{R}}\right) e^{-a_{\Lambda} \bar{R}}\right. \\
& +\frac{\gamma}{5}\left[\frac{1}{\left[\frac{9}{4}-(\sqrt{\gamma}+1)^{2} a_{\Lambda}^{2}\right]^{3}}\left[\frac{1}{\bar{R}^{2}}+\frac{(\sqrt{\gamma}+1) a_{\Lambda}}{\bar{R}}\right] e^{-(\sqrt{\gamma}+1) a_{\Lambda} \bar{R}}\right. \\
& -\frac{1}{\left[\frac{9}{4}-(\sqrt{\gamma}+3)^{2} a_{\Lambda}^{2}\right]^{3}}\left[\frac{1}{\bar{R}^{2}}+\frac{(\sqrt{\gamma}+3) a_{\Lambda}}{\bar{R}}\right] e^{-(\sqrt{\gamma}+3) a_{\Lambda} \bar{R}} \\
& -\frac{1}{\left(\frac{9}{4}-a_{\Lambda}^{2}\right)^{3}}\left(\frac{1}{\bar{R}^{2}}+\frac{a_{\Lambda}}{\bar{R}}\right) e^{-a_{\Lambda} \bar{R}} \\
& \left.\left.+\frac{1}{\left(\frac{9}{4}-9 a_{\Lambda}^{2}\right)^{3}}\left(\frac{1}{\bar{R}^{2}}+\frac{3 a_{\Lambda}}{\bar{R}}\right) e^{-3 a_{\Lambda} \bar{R}}\right]\right\}
\end{aligned}
$$

where $a_{\Lambda}\left(\equiv a_{Z} / \Lambda\right)$ is the scaled reciprocal Debye length and $\bar{R} \equiv R / a_{Z}$. To describe the motion of the high-energy projectile electron, we assume that it is moving on a straight-line trajectory in the so-called natural coordinate frame (Jung and Cho 1995) in which the axis of quantization $z$ is chosen perpendicular to the collision plane. Then, the position of the projectile electron can be written as a function of time $t$ and the impact parameter $b$ :

$$
\mathbf{R}(t)=v t \hat{\mathbf{x}}+b \hat{\mathbf{y}}
$$


where $v$ is the velocity of the projectile electron. Here, $t=0$ is arbitrarily chosen as the instant at which the projectile electron makes its closest approach to the target ion. Under these circumstances, in the $1 s \rightarrow 2 p$ excitation, a conservation law prohibits the $1 s \rightarrow 2 p_{0}(m=0)$ transition; only the $m= \pm 1$ substates $\left(2 p_{ \pm 1}\right)$ of the $2 p$ level are possible. In this natural coordinate frame, i.e., the collision plane (the $(x, y)$ plane) perpendicular to the quantization axis (the $z$ axis), the spherical harmonic $Y_{1 \pm 1}^{*}(\hat{R})$ is represented as

$$
Y_{1 \pm 1}^{*}(\hat{R})=\mp \sqrt{\frac{3}{8 \pi}} \frac{(\bar{v} t \mp i \bar{b})}{\bar{R}},
$$

where $\bar{v} \equiv v / a_{Z}$ and $\bar{b}\left(\equiv b / a_{Z}\right)$ is the scaled impact parameter. Then, the $1 s \rightarrow 2 p_{ \pm 1}$ transition amplitudes $T_{2 p_{ \pm 1}, 1 s}$ are found to be

$$
\begin{aligned}
& T_{2 p_{ \pm 1}, 1 s}=T_{2 p_{ \pm 1}, 1 s}\left(\bar{b}, a_{\Lambda}, \gamma, \bar{E}\right) \\
&=\mp \frac{12}{1+c(\gamma)} \frac{e^{2}}{\hbar v} \int_{0}^{\infty} d \tau(\tau \sin \beta \tau \mp \bar{b} \cos \beta \tau) \\
& \times\left\{\frac{1}{\left(\frac{9}{4}-a_{\Lambda}^{2}\right)^{3}}\left[\frac{1}{\left(\tau^{2}+\bar{b}^{2}\right)^{3 / 2}}+\frac{a_{\Lambda}}{\tau^{2}+\bar{b}^{2}}\right] e^{-a_{\Lambda}\left(\tau^{2}+b^{2}\right)^{1 / 2}}\right. \\
&+ \frac{\gamma}{5}\left[\frac{1}{\left[\frac{9}{4}-(\sqrt{\gamma}+1)^{2} a_{\Lambda}^{2}\right]^{3}}\left[\frac{1}{\left(\tau^{2}+\bar{b}^{2}\right)^{3 / 2}}+\frac{(\sqrt{\gamma}+1) a_{\Lambda}}{\tau^{2}+\bar{b}^{2}}\right] e^{-(\sqrt{\gamma}+1) a_{\Lambda}\left(\tau^{2}+\bar{b}^{2}\right)^{1 / 2}}\right. \\
&-\frac{1}{\left[\frac{9}{4}-(\sqrt{\gamma}+3)^{2} a_{\Lambda}^{2}\right]^{3}}\left[\frac{1}{\left(\tau^{2}+\bar{b}^{2}\right)^{3 / 2}}+\frac{(\sqrt{\gamma}+3) a_{\Lambda}}{\tau^{2}+\bar{b}^{2}}\right] e^{-(\sqrt{\gamma}+3) a_{\Lambda}\left(\tau^{2}+\bar{b}^{2}\right)^{1 / 2}} \\
&\left.\left.-\frac{1}{\left(\frac{9}{4}-a_{\Lambda}^{2}\right)^{3}}\left[\frac{1}{\left(\tau^{2}+\bar{b}^{2}\right)^{3 / 2}}+\frac{a_{\Lambda}}{\tau^{2}+\bar{b}^{2}}\right] e^{-a_{\Lambda}\left(\tau^{2}+\bar{b}^{2}\right)^{1 / 2}}\right]\right\} \\
&\left.\left.+\frac{1}{\left(\frac{9}{4}-9 a_{\Lambda}^{2}\right)^{3}}\left[\frac{1}{\left(\tau^{2}+\bar{b}^{2}\right)^{3 / 2}}+\frac{3 a_{\Lambda}}{\tau^{2}+\bar{b}^{2}}\right] e^{-3 a_{\Lambda}\left(\tau^{2}+\bar{b}^{2}\right)^{1 / 2}}\right]\right\}
\end{aligned}
$$

where $\tau\left(\equiv v t / a_{Z}\right)$ is the dimensionless time, $\beta\left(\equiv \omega_{2 p_{ \pm 1}, 1 s} a_{Z} / v\right)=3 / 8 \sqrt{\bar{E}}$, $\bar{E}\left(\equiv m v^{2} / 2 Z^{2} R y\right)$ is the scaled kinetic energy of the incident electron, and $R y\left(=m e^{4} / 2 \hbar^{2} \approx 13.6 \mathrm{eV}\right)$ is the Rydberg constant.

\section{Orientation parameter}

The orientation parameter for the $1 s \rightarrow 2 p_{ \pm 1}$ excitations is defined as

$$
L_{\perp}\left(\bar{b}, a_{\Lambda}, \gamma, \bar{E}\right)=\frac{\left|T_{2 p_{+1}, 1 s}\left(\bar{b}, a_{\Lambda}, \gamma, \bar{E}\right)\right|^{2}-\left|T_{2 p_{-1}, 1 s}\left(\bar{b}, a_{\Lambda}, \gamma, \bar{E}\right)\right|^{2}}{\left|T_{2 p_{+1}, 1 s}\left(\bar{b}, a_{\Lambda}, \gamma, \bar{E}\right)\right|^{2}+\left|T_{2 p_{-1}, 1 s}\left(\bar{b}, a_{\Lambda}, \gamma, \bar{E}\right)\right|^{2}},
$$

where $\left|T_{2 p_{ \pm 1}, 1 s}\left(\bar{b}, a_{\Lambda}, \gamma, \bar{E}\right)\right|^{2}$ are the $1 s \rightarrow 2 p_{ \pm 1}$ transition amplitudes. This quantity $L_{\perp}\left(\bar{b}, a_{\Lambda}, \gamma, \bar{E}\right)$ is a measure of the expectation value of the orbital angular momentum transferred to the bound electron in the target ion owing to the direct $1 s \rightarrow 2 p_{ \pm 1}$ excitations. Since the line-intensity ratios are directly related to the $1 s \rightarrow 2 p_{ \pm 1}$ excitation rates, the orientation parameter is connected to the relative number of coincidences for RHC (right-hand circularly polarized) and LHC (left-hand circularly polarized) photons emitted owing to radiative decay from the excited $2 p_{-1}$ and $2 p_{+1}$ states to the ground $1 s$ state. Then, the nonideal-plasma 

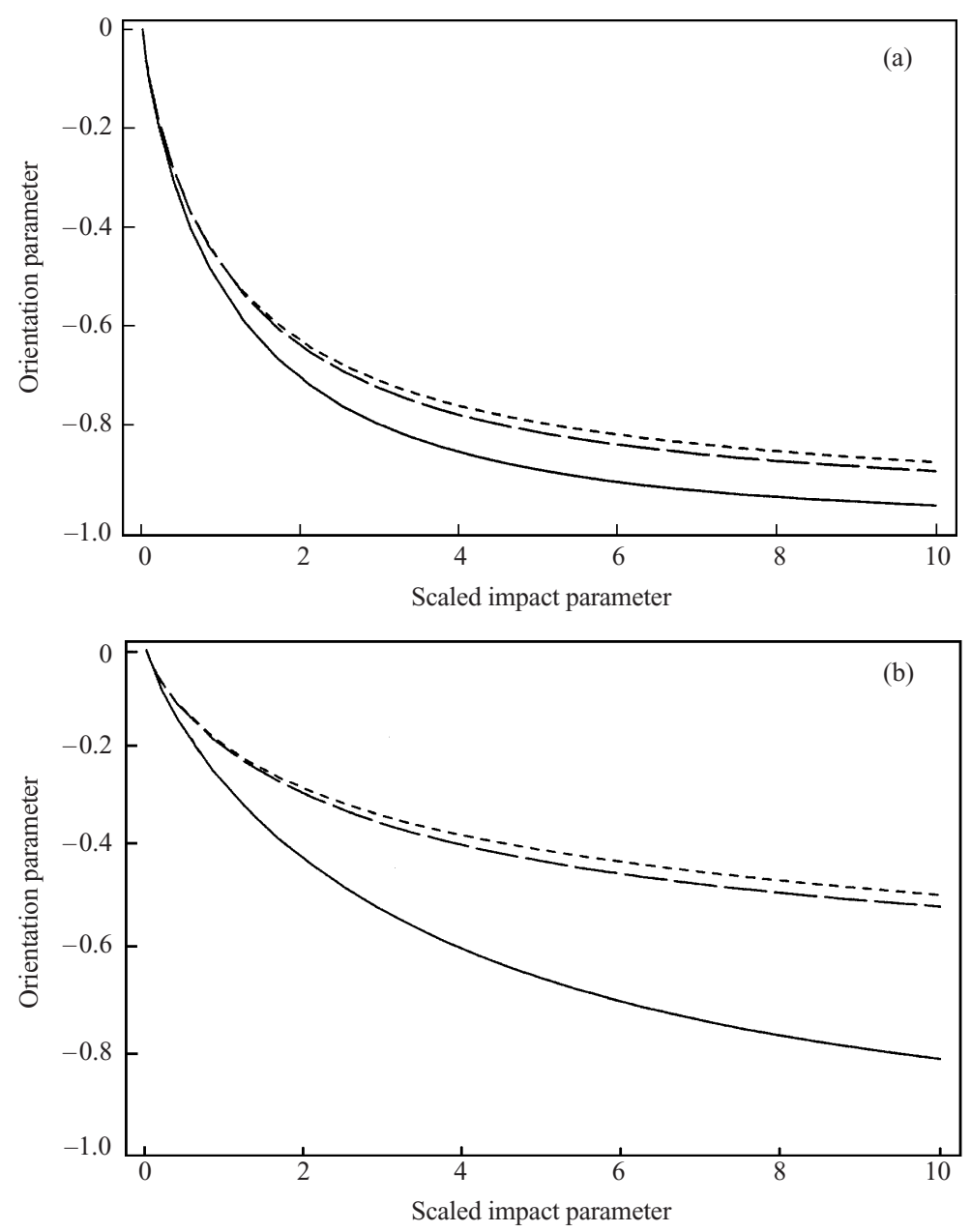

Figure 1. The orientation parameter $L_{\perp}\left(\bar{b}, a_{\Lambda}, \gamma, \bar{E}\right)$ for $a_{\Lambda}=0.1$ as a function of the scaled impact parameter $\bar{b}\left(=b / a_{Z}\right):-, \gamma=0$ (ideal case); ---, $\gamma=0.1$ (weakly nonideal case); $\ldots \ldots, \gamma, \gamma=1$ (strongly nonideal case); (a) $\bar{E}=9$; (b) $\bar{E}=81$.

effects due to the properties of the plasma change the relative number of coincidences for RHC and LHC photons. Thus, orientation phenomena could be exploited as a plasma diagnostic.

In order to explicitly investigate plasma-screening and collective effects on the orientation parameter and the propensity rule for direct electron-ion excitations, we consider a hydrogenic-ion target with $a_{\Lambda}=0.1$. Here, we consider ideal, weakly, and strongly nonideal cases: $\gamma=0, \gamma=0.1$, and $\gamma=1$, and two cases of projectile energies: $\bar{E}=9$ and $\bar{E}=81$, since the semiclassical straight-line trajectory method is known to be valid for high projectile energies $\bar{E}>7$ (Bethe and Jackiw 1986). Figure 1 shows the orientation parameter $L_{\perp}\left(\bar{b}, a_{\Lambda}, \gamma, \bar{E}\right)$ for direct $1 s \rightarrow 2 p_{ \pm 1}$ excitations as a function of the impact parameter, the nonideal-plasma parameter, the Debye length, and the energy of the projectile electron. Because of the propensity rule, the probability of populating the $2 p_{-1}$ state dominates the probability of populating the $2 p_{+1}$ state in planar collisions. The result shows that the nonideal 
effect significantly reduces the propensity of the $1 s \rightarrow 2 p_{-1}$ transition. It is found that the nonideal effect, i.e., the collective effect, on the orientation parameter increases with increasing projectile energy. It is also found that the propensity of the $1 s \rightarrow 2 p_{-1}$ transition is significantly suppressed with increasing energy of the projectile electron. Thus, the variation of the propensity of the $1 s \rightarrow 2 p_{-1}$ excitation due to the nonideal effect could be used in plasma diagnostics to determine the nonideality of a plasma.

\section{Summary and discussion}

In this paper, we have investigated plasma-screening and collective effects on orientation phenomena for direct electron-hydrogenic-ion collisions in a classical nonideal plasma. An effective pseudopotential model taking into account plasmascreening and collective effects was applied to describe the projectile-target interaction potential in a nonideal plasma. The semiclassical straight-line trajectory method was applied to describe the motion of the projectile electron, and the target-wave system was represented by quantum-mechanical wave functions. The orientation parameter $L_{\perp}$ for direct $1 s \rightarrow 2 p_{ \pm 1}$ excitations was obtained as a function of the scaled impact parameter $\bar{b}$, the nonideal-plasma parameter $\gamma$, the scaled reciprocal Debye length $a_{\Lambda}$, and the scaled projectile energy $\bar{E}$. The result shows that the probability of populating the $2 p_{-1}$ state dominates the probability of populating the $2 p_{+1}$ state in planar collisions. It should be noted that the nonideal effect, i.e., the collective effect, reduces the propensity of the $1 s \rightarrow 2 p_{-1}$ transition. It was also found that the nonideal effect on the orientation parameter increases with increasing energy of the projectile electron. These results provide a general description of orientation phenomena for $s-p$ excitations in a classical nonideal plasma.

\section{Acknowledgements}

The author acknowledges Professor R. J. Gould and Professor T. M. O'Neil for warm hospitality while visiting the University of California, San Diego. The author thanks Professor B. Talin and Professor R. Sauerbrey for useful discussions on nonideal plasmas. He also thanks Professor W. Ebeling for providing him with useful references. The author would like to thank the anonymous referee for suggesting improvements to this text.

This work was supported by the Korean Ministry of Education through the Brain Korea (BK21) Project, by Hanyang University, South Korea, made in the program year of 2001, and by the Korea Basic Science Institute through the HANBIT User Development Program (FY2002).

\section{References}

Andersen, N., Dowek, D., Dubois, A., Hansen, J. P. and Nielsen, S. E. 1990 Physica Scripta 42,266 .

Arfken, G. B. and Weber, H. J. 2000 Mathematical Methods for Physicists, 5th edn. New York: Academic Press.

Arkhipov, Yu. V. and Davletov, A. E. 1998 Phys. Lett. 247A, 339.

Arkhipov, Yu. V., Baimbetov, F. B. and Davletov, A. E. 2000a Eur. Phys. J. D8, 299.

Arkhipov, Yu. V., Baimbetov, F. B., Davletov, A. E. and Starikov, K. V. 2000b Plasma Phys. Contr. Fusion 42, 455. 
Baimbetov, F. B., Nurekenov, Kh. T. and Ramazanov, T. S. 1995 Phys. Lett. 202A, 211.

Balashov, V. V., Grun-Grzhimailo, A. N. and Kabachnik, N. M. 2000 Polarization and Correlation Phenomena in Atomic Collisions. Dordrecht: Kluwer.

Bethe, H. A. and Jackiw, R. 1986 Intermediate Quantum Mechanics. New York: Benjamin.

Bethe, H. A. and Salpeter, E. E. 1957 Quantum Mechanics of One-and Two-Electron Atoms. Berlin: Springer-Verlag.

Janev, R. K., Presnyakov, L. P. and Shevelko, V. P. 1985 Physics of Highly Charged Ions. Berlin: Springer-Verlag.

Jung, Y.-D. 1993 Astrophys. J. 409, 841.

Jung, Y.-D. 1995a Phys. Plasmas 2, 332.

Jung, Y.-D. 1995b Phys. Plasmas 2, 987.

Jung, Y.-D. and Cho, I.-D. 1995 Phys. Rev. E52, 5333.

Kobzev, G. A., Iakubov, I. T. and Popovich, M. M. 1995 Transport and Optical Properties of Nonideal Plasma. New York: Plenum.

McDowell, M. R. C. and Coleman, J. P. 1970 Introduction to the Theory of Ion-Atom Collisions. Amsterdam: North-Holland. 